\title{
Ecología y conservación de los bosques montanos de Polylepis. Una introducción al número especial
}

\author{
Griet A. E. CuYCKens ${ }^{1,2} \&$ DANiel Renison ${ }^{3,}$ 区 \\ ${ }^{1}$ Centro de Estudios Territoriales Ambientales y Sociales (CETAS). San Salvador de Jujuy, Jujuy, Argentina. ${ }^{2}$ Instituto de \\ Ecorregiones Andinas (CONICET - Universidad Nacional de Jujuy). ${ }^{3}$ Instituto de Investigaciones Biológicas y Tecnológicas, \\ Centro de Ecología y Recursos Naturales Renovables (CONICET - Universidad Nacional de Córdoba). Córdoba, Argentina.
}

\begin{abstract}
Resumen. El género Polylepis pertenece a la familia Rosaceae e incluye arbustos y árboles que crecen en las altas montañas de Los Andes y de las Sierras Grandes de Córdoba. En su mayoría, forma bosques cuya canopia es dominada por una especie del género Polylepis. Se caracteriza por tener una corteza con muchas láminas color rojizo, ramas retorcidas, hojas pequeñas compuestas y flores inconspicuas, y por crecer - por necesidad o aptitud de hábitat - mayormente en quebradas rocosas. En términos evolutivos, es probable que se haya generado a partir del género Acaena por poliploidía. Los bosques de Polylepis proveen servicios ambientales y socioeconómicos de gran valor, como la protección de fuentes de agua, el control de la erosión y la regulación del microclima y del hábitat para numerosas especies. Los árboles de Polylepis poseen importancia económica para las comunidades campesinas. En este número especial presentamos algunos de los últimos avances en el estudio de los bosques de Polylepis. Muchos de los trabajos fueron presentados durante el último Congreso de Ecología y Conservación de Polylepis que se realizó en Jujuy, Argentina, en el año 2016. Esperamos que el próximo congreso, que tendrá lugar en Ecuador, cuente con un número creciente de estudios y participantes.
\end{abstract}

[Palabras clave: Sudamérica, Andes, distribución, revisión cuantitativa]

\begin{abstract}
Aвstract. Ecology and conservation of Polylepis montane forests. An introduction to the special issue. The genus Polylepis belongs to the Rosaceae family and includes shrubs and trees that grow in the high mountains of the Andes and the Sierras Grandes of Córdoba. It forms forests with a canopy mainly dominated by one species of the genus Polylepis. The genus is characterized by paper-thin layers of rust-red bark, sinuous trunks and branches, tiny compound leaves and inconspicuous flowers; it is found mostly in rocky canyons because of necessity or habitat aptitude. Evolutionarily, it was probably originated from the genus Acaena by polyploidy. The Polylepis forests provide valuable environmental and socio-economic services, such as the protection of water sources, erosion control and regulation of the microclimate, and offers habitat for numerous species. The Polylepis trees have economic importance for the peasant communities. In this special issue, we present some of the latest advances in the study of Polylepis. Many of them were presented during the last meeting of Ecology and Conservation of Polylepis, held in Jujuy, Argentina, in 2016. We hope that the next meeting (to be held in Ecuador) will have an increasing number of studies and participants.
\end{abstract}

[Keywords: South America, Andes, distribution, quantitative review]

\section{INTRODUCCIÓN}

\section{Los bosques y arbustales de Polylepis}

Los bosques y arbustales del género Polylepis forman pequeños parches comúnmente presentes en laderas de montañas y quebradas rocosas a lo largo de Los Andes y montañas contiguas (Arnal et al. 2014). Con unos $5400 \mathrm{~km}$ desde su extremo norte hasta el sur, los bosques de Polylepis son una de las comunidades boscosas con mayor extensión latitudinal del mundo, ya que abarcan desde Venezuela hasta sectores de las Sierras Grandes de Córdoba, en la Argentina (Figura 1). En muchos lugares forman el límite superior del bosque continuo. A nivel mundial, Polylepis tarapacana es la especie de porte arbóreo que llega a mayor altitud sobre el nivel del mar
(Cuyckens et al. 2016). Son árboles y arbustos que suelen dominar la canopia de bosques y arbustales. Por ello, las formaciones boscosas donde se encuentran las especies de Polylepis toman su nombre. Lo que sabemos y lo que ignoramos sobre la ecología y la conservación de los bosques y arbustales de Polylepis fue revisado por Renison et al. (2018). Las especies de Polylepis conforman la familia botánica Rosaceae, tribu Sanguisorbeae. Se los llama comúnmente queñoa (del runasimi, kiwiña), coloradita (Colombia), tabaquillo (Argentina) o árbol de papel (Ecuador), entre otros. Por su etimología, el nombre deriva de "poly" muchos y "lepides", láminas (Muñoz-Schick et al. 2012), dado que posee muchas láminas finas en el tronco. Aún se desconoce la función de estas láminas, pero se estima que brindan protección contra el frío y el fuego. La corteza 


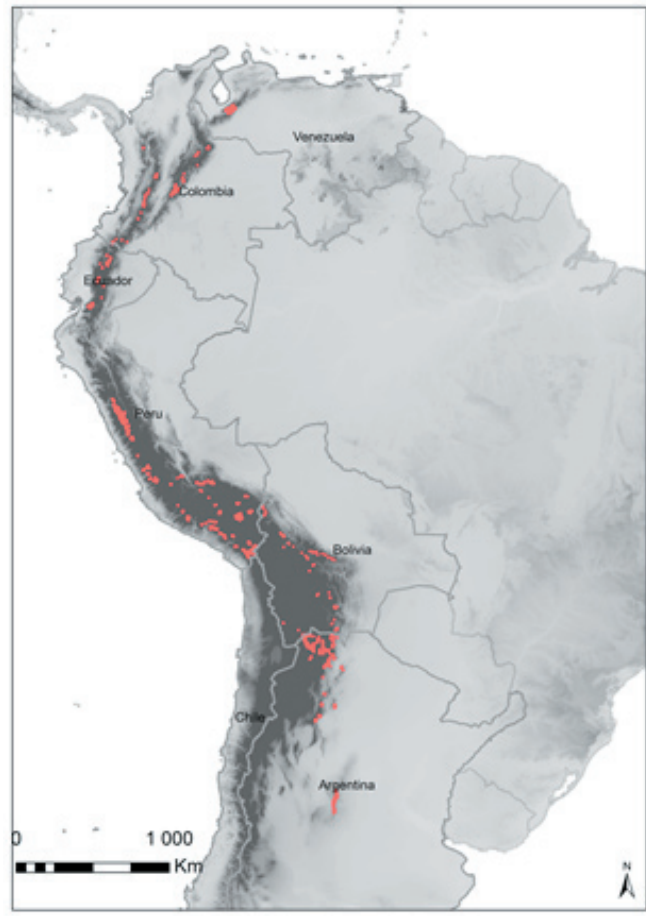

Figura 1. Mapa de la distribución puntual del género Polylepis a lo largo de Los Andes y en las Sierras Grandes de Córdoba (Argentina).

Figure 1. Map of distribution records of the genus Polylepis along the Andes and in the Sierras Grandes of the Córdoba province in Argentina.

es de color rojizo y se encuentra en troncos retorcidos; las ramas también son retorcidas y caídas, lo cual les brinda a los árboles su aspecto característico (Figura 2a). El follaje es siempre verde o semideciduo según la especie, y de color verde azulado o grisáceo, lo que facilita el reconocimiento de los bosques o individuos de Polylepis en imágenes satelitales. Las hojas son pequeñas y compuestas, imparipinnadas, y tienen entre 3 y 9 folíolos según la especie. Las flores, hermafroditas, son inconspicuas y dispuestas en racimos. Los frutos son aquenios duros con tres alas (Figura 2b). En toda la tribu Sanguisorbeae, las flores son polinizadas principalmente por el viento (anemofilia), lo cual se considera un evento evolutivo relevante en la adaptación a las tierras altas, donde los insectos son más escasos en relación con lugares de climas cálidos y de menor altitud (Fjeldså y Kessler 2004), aunque se pueden observar también visitantes florales como abejas. El polen del género Polylepis es mónada, con una forma isopolar, relativamente esférico, tricolporado, pontoperculado y achatado ligeramente en los polos (Simpson 1986; Chung et al. 2010). En este número se presenta un trabajo sobre palinología que intenta responder la siguiente pregunta: ¿es posible inferir el nivel de ploidía de especies de Polylepis mediante una relación del número cromosómico y el tamaño del genoma con datos morfométricos de células guardianas estomáticas o granos de polen? utilizando diferentes especies de Ecuador (Caiza et al. 2018). La taxonomía del género Polylepis es compleja y los últimos avances fueron expuestos por Segovia-Salcedo et al. (2018).

\section{Origen}

Evolutivamente, el género Polylepis se encuentra cercano al género Acaena (tribu Sanguisorbeae). Existe la hipótesis que Polylepis se originó a través de un proceso de hibridación alopoliploide entre Acaena elongata y Acaena cylindristachya (Kerr 2004), dos especies de Acaena que aún comparten su hábitat con Polylepis quadrijuga en la Cordillera Oriental colombiana. La contribución a este número sobre la distribución de ésta y las otras especies presentes en Colombia representa un aporte a esta cuestión (Fajardo-Gutiérrez

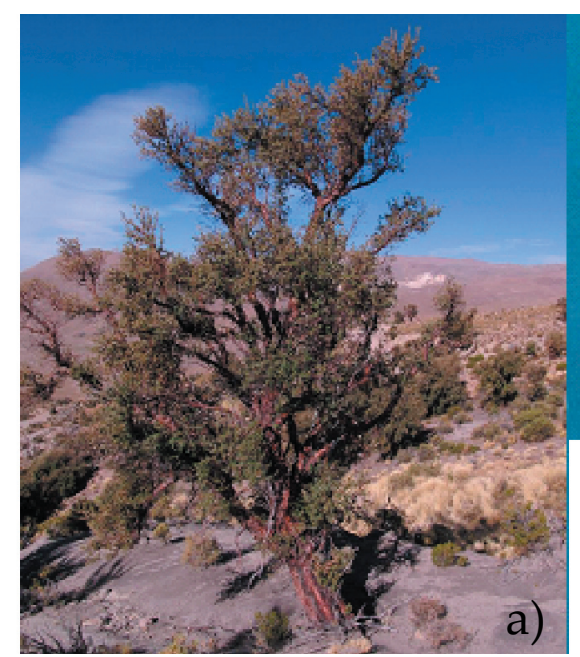

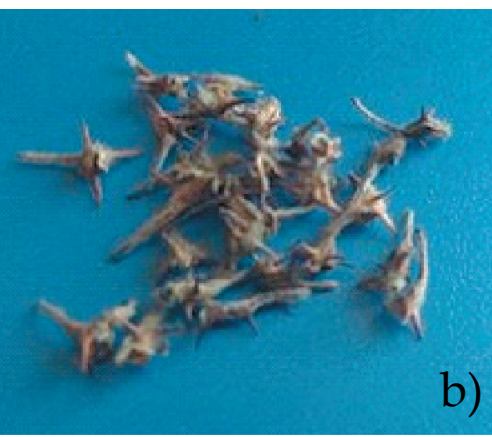

Figura 2. a) Árbol de Polylepis tomentella en el que se puede observar su corteza característica (foto: F. Mazzini) y b) detalle de los frutos (aquenios) trialados de Polylepis hieronymi.

Figure 2. a) Polylepis tomentella tree in which its characteristic bark can be observed (photo: F. Mazzini) and b) detail of the trialated fruits (achenes) of Polylepis hieronymi. 
et al. 2018). La poliploidía es el resultado de un proceso de hibridación y se define por la posesión de varios juegos completos de cromosomas. Representa un mecanismo evolutivo clave en el proceso de especiación, en especial en angiospermas (Soltis et al. 2015; Wendel 2015). Se la define como autoploide cuando surge a partir de una hibridación en la misma especie o aloploide cuando ocurre entre diferentes especies (Leitch and Bennett 1997), como sería el caso de Polylepis. La poliploidía en el género Polylepis está poco estudiada, pero en este número se incluyen nuevos avances sobre su manifestación el género en Ecuador (Caiza et al. 2018).

\section{Biodiversidad y endemismos}

Los bosques nativos tienden a albergar más biodiversidad que los bosques conformados principalmente por especies exóticas (Southwood 1961; Harding and Rose 1986). En particular, los bosques de Polylepis ofrecen hábitat a numerosas especies en lugares muchas veces áridos o despoblados de árboles. Gran cantidad de especies animales hacen uso de sus recursos como alimento o refugio. Por ejemplo, en los bosques de Polylepis de Perú, Bolivia y la Argentina se encontró al murciélago Lasiurus cinereus (L. Aguirre comunicación personal) (Figura 3). Son ecosistemas que albergan una biota única, en los que se estudiaron con especial interés las aves especialistas de hábitat o con altos niveles de endemismo. Sevillano-Ríos et al. (2018) brinda una revisión sobre la ecología y conservación de las aves asociadas a los bosques de Polylepis. Sin embargo, aún falta mucha información sobre la biodiversidad y los endemismos de los bosquecillos de Polylepis, que puede ser particularmente rica, como lo demuestra la contribución sobre insectos coleópteros que habitan los bosques de Polylepis (Rossi et al. 2018).

\section{Regulación hídrica}

Los bosques de Polylepis protegen las fuentes de agua (Fjeldså and Kessler 1996), regulan la escorrentía y mejoran la captación de agua. Poca et al. (2018) muestran, para el centro de Argentina, cómo la degradación de los bosques de Polylepis reduce la capacidad de retener el agua en el suelo. Dado que los días con neblina son comunes en muchas de las montañas donde habita Polylepis (Figura 4), estos bosques aumentarían el aporte hídrico al actuar como una red de hojas y ramas donde se nuclea la neblina. Además, los árboles, la

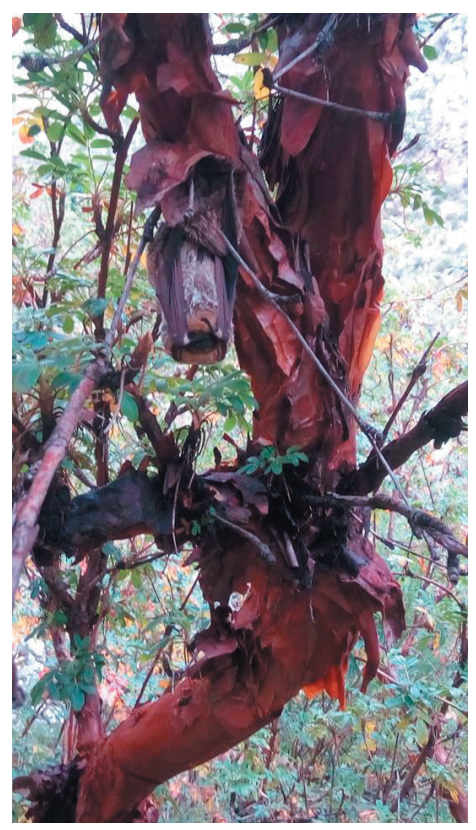

Figura 3. Murciélago Lasiurus cinereus en tabaquillo $P$. australis en Córdoba (Argentina) (Foto: M. V. Sánchez Dománguez).

Figure 3. Bat Lasiurus cinereus in P. australis in Córdoba (Argentina) (Photo: M. V. Sánchez Dománguez).

vegetación asociada y los suelos en buena condición mejoran la calidad del agua que alcanza los ríos y arroyos, y hace más económica su potabilización para el consumo humano. Es por ello que la conservación y la restauración de los bosques de Polylepis podrían mejorar el aporte hídrico y reducir los costos que implica potabilizar el agua. De todos modos, aún faltan estudios que ilustran este aspecto.

\section{Suelos y captura de carbono}

Los bosques de Polylepis reducen la erosión de los suelos y retienen sedimentos y nutrientes (Fjeldså and Kessler 2004; Renison et al. 2010). Mediante las raíces, las hojas y las ramas que caen, los árboles proveen materia orgánica a los suelos, fertilizándolos y aumentando su volumen y su capacidad de absorción de agua. Se forman suelos fértiles que aumentan la productividad primaria y la productividad agrícola y ganadera que se realiza en los parches entre los bosques. Los suelos en los bosques de Polylepis se caracterizan por tener un $\mathrm{pH}$ ácido, textura franco-arenosa, niveles de materia orgánica elevados y una limitada disponibilidad de nutrientes (Fernández et al. 2018). En los bosques y sus suelos se captura gran cantidad de carbono atmosférico, lo que contribuye a mitigar el impacto de la quema de combustible fósil (Aranibar 2015). Esto 


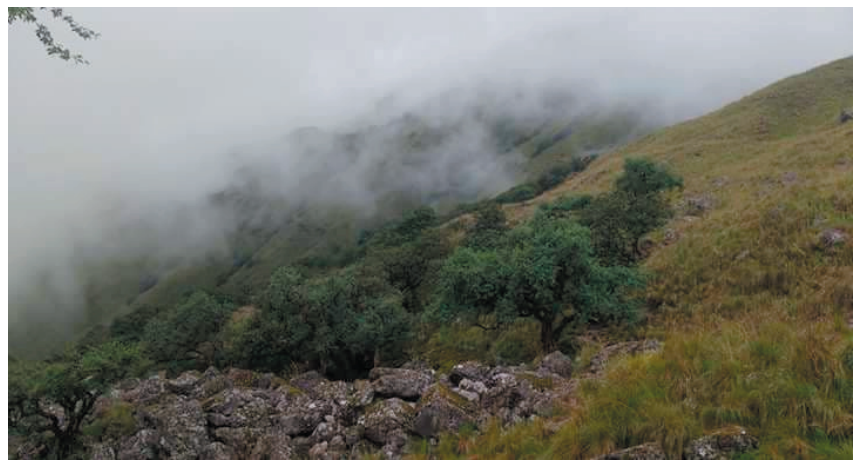

Figura 4. Bosque de Polylepis australis capturando neblina en el parque provincial Potrero de Yala (Jujuy, Argentina).

Figure 4. Polylepis australis forest capturing haze in the Potrero de Yala Provincial Park (Jujuy, Argentina).

cobra especial importancia en los bosques de Polylepis, ya que muchas veces son el único exponente arbóreo en el ambiente que ocupan. En este número especial sobre Polylepis encontramos un artículo sobre las características morfológico-funcionales, la diversidad arbórea y la tasa de crecimiento y de secuestro de carbono en especies y ecosistemas de Polylepis del sur de Ecuador (Montalvo et al. 2018).

\section{Turismo}

Los bosques aumentan la calidad paisajística de la zona y ofrecen un mejor producto al turismo. Existen iniciativas turísticas directamente orientadas hacia los bosques de Polylepis o hacía las aves que allí habitan (e.g., https://goo.gl/oe6AAG, https://goo.gl/ PPEH21 o https://goo.gl/wfvztx). Conocemos un solo estudio en el que se evalúa el efecto del turismo sobre los bosques de Polylepis o sobre su biota asociada (Heil et al. 2007), por lo que se necesitarían más estudios que brinden información útil para ordenar y reducir el impacto del turismo. Otros emprendimientos turísticos no sólo orientan a los visitantes hacía los bosques, sino que también intentan restaurar bosques de Polylepis (https://goo.gl/ 3aP7nG).

\section{Usos directos}

Los bosques de Polylepis son una fuente fundamental de madera como combustible para la cocción de alimentos. Se los usa para fabricar artesanías y confeccionar postes, vigas (Figura 5a y b) y herramientas. Las láminas del tronco tienen un uso medicinal: se hace una infusión color café que ayudaría a liberar las vías respiratorias y curaría enfermedades renales; además, sirve para teñir tejidos. En su composición química se encontraron varios ácidos (e.g., el ácido pomólico, principal componente citotóxico en el tallo y en la corteza de Polylepis racemosa. Los habitantes locales usan la corteza para tratar la inflamación y el cáncer uterino (Neto et al. 2000). Asimismo, los bosques son zonas utilizadas para el pastoreo del ganado doméstico nativo (llamas, alpacas) e introducido (ovejas y vacas) Renison et al. (2013).

\section{Amenazas}

Desde tiempos precolombinos, los bosques de Polylepis han sido un hábitat muy intervenido y fragmentado por las actividades humanas (Fjeldså and Kessler 1996; Purcell and Brelsford 2004). Etter y Villa (2000) citan una pérdida de entre 70 y $90 \%$ de la cobertura original para la Cordillera Oriental colombiana. Se calcula que $98 \%$ de los bosque de Polylepis desapareció de las regiones de Perú (Fjeldså and Kessler 1996); por su parte, en Bolivia se perdió 50\% en la Cordillera Occidental y 99\% en regiones de los Andes Orientales En consecuencia, es

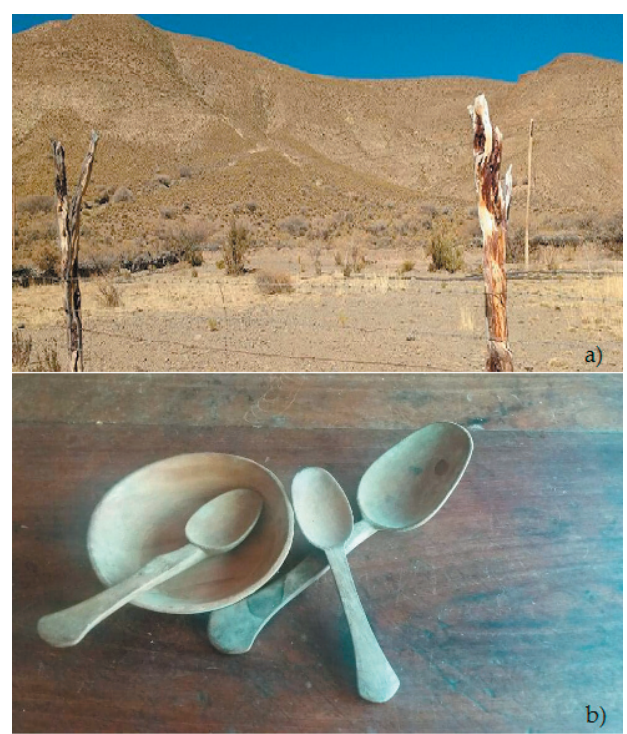

Figura 5. Uso de Polylepis tomentella como a) postes para la construcción de corrales (Quebraleña, Jujuy, Argentina) y b) para la elaboración de utensilios (Quebraleña, Jujuy, Argentina) (Foto: M. F. Galian, maestro de Quebraleña).

Figure 5. Use of Polylepis tomentella as a) poles for the construction of corrals (Quebraleña, Jujuy, Argentina) and $b$ ) for the preparation of tools (Quebraleña, Jujuy, Argentina) (Photo: M. F. Galian, Quebraleña teacher). 
uno de los ecosistemas más afectados de las zonas altoandinas (Navarro et al. 2005).

Entre sus amenazas encontramos la expansión de la frontera agrícola, la tala y el uso para leña, el reemplazo o la invasión por especies exóticas, el cambio climático, la minería, la expansión de carreteras y las prácticas ganaderas. Entre las prácticas ganaderas se pueden mencionar la quema para estimular el rebrote de los pastos, el ramoneo directo y el pisoteo por el ganado doméstico. En un caso de estudio, la implantación de especies exóticas alteró los efectos naturales de los árboles maternos sobre el micrositio y la producción de semillas, como ilustra el estudio de P. subtusalbida en Cochabamba, Bolivia (Gareca et al. 2018).

\section{Número especial}

Este número especial fue ideado en el IV Congreso de Ecología y Conservación de Polylepis que tuvo lugar en Jujuy (Argentina) en septiembre del 2016. Muchos de los trabajos de este número fueron allí presentados en forma oral y otros son nuevos. Sin embargo, la idea de publicar los avances en las investigaciones de Polylepis surgió mucho antes, durante los I, II y III congresos que tuvieron lugar en Cochabamba (Bolivia), Cusco (Perú) y Arica (Chile), respectivamente. Este número abarca varios aspectos científicos del género Polylepis, desde lo taxonómico y lo ecológico, e incluye aspectos de dendrocronología (Lanza et al., este número). Se tienen en cuenta varias especies ( $P$. australis, $P$. incana, $P$. incarum, $P$. lanuginosa, P. microphylla, P. neglecta, P. pacensis, $P$. pauta, $P$. quadrijuga, $P$. racemosa, $P$. reticulata, $P$. sericea, $P$. subtusalbida, $P$. tomentella y $P$. weberbaueri) de diferentes países a lo largo de su distribución (Argentina, Bolivia, Colombia, Ecuador y Perú) y también se incluyen trabajos de revisión.

\section{Conclusiones}

Por su importancia en la alta montaña y por sus actuales amenazas, los bosques y arbustales de Polylepis merecen recibir acciones de conservación, además de un adecuado manejo y aprovechamiento. A pesar de que los esfuerzos han ido creciendo, sobre todo en algunas regiones (ver Renison et al. 2018), aún existen vastas regiones con un pobre conocimiento y también ciertas ramas de conocimiento básico que todavía no fueron cubiertas como, por ejemplo, la fenología y o la historia natural. Estudiar la fenología reproductiva podría apoyar a los programas de conservación y reforestación al identificar los meses ideales para colectar semillas y las características alométricas de las plantas semilleras (Henríquez et al. 2012). Conocer su comportamiento germinativo aporta al conocimiento sobre la producción de plantines para actividades de reforestación. Con respecto a la fenología, en este número incluimos un trabajo sobre $P$. incarum en La Paz (Bolivia) (Lopez et al. 2018) y un trabajo relevante para el conocimiento de la reproducción sexual de tres especies de Polylepis en Bolivia (Vega et al. 2018).

A pesar de estos vacíos de conocimiento, la ciencia que estudia a los bosques de Polylepis o "polylepología" fue creciendo desde el primer congreso sobre su conservación y ecología. Las reuniones con los expertos y los experimentos en el rubro también ponen de manifiesto la falta de una puesta en común sobre la recuperación o la restauración de los bosques de Polylepis (Morales et al. 2018). Alentamos a los jóvenes a incursionar en la "polylepología" para enriquecerla y completarla, como así también apostamos a las futuras generaciones de políticos y tomadores de decisión para que tengan la visión y el coraje suficiente para embarcarse en la protección y el manejo de las especies a lo largo de todo el rango de distribución del género, con acciones en común.

Agradecimientos. A los sucesivos organizadores de los congresos de Ecología y Conservación de bosques de Polylepis, quienes en cuatro ocasiones permitieron madurar las ideas reflejadas en el presente número especial. A Esteban Jobbágy, en su función de editor de Ecología Austral, por estimular que el número especial de Polylepis se realice en dicha revista. A Eva Laura Florio, asistente editorial por la paciencia durante la edición de la revista. A los numerosos revisores que aportaron tiempo y conocimiento para mejorar los manuscritos que integran el tomo especial $\mathrm{y}$, desde ya, a todos los autores que trabajaron arduamente para avanzar en el conocimiento sobre los bosques de Polylepis.

\section{ReFERENCIAS}

Aranibar, L. F. M. 2015. Estado actual del bosque de Polylepis y su eficiencia en la captura de $\mathrm{CO}_{2}$ en la provincia Tarata, departamento de Tacna. Revista Ciencia \& Desarrollo 19: 36-43.

Arnal, H., A. Sampson, G. Navarro, et al. 2014. Mapa pan Andino de bosques de Polylepis prioritarios para la conservación. Disponible en: <http:// condesan.org/portal/mapa-pan-andino-de-bosques-de-polylepis-prioritarios- 
para-conservacion-american-bird-conservancy> Consultado: 09/2016.

Caiza, J. C., D. Vargas, C. Olmedo, M. arboleda, L. Boada; O. Acurio, C. Arroyo, A. Debut y M. Segovia-Salcedo. 2018. Morfometría y morfología de estomas y de polen como indicadores indirectos de poliploidía en especies del género Polylepis (Rosaceae) en Ecuador. Ecología Austral 28: 175-187

Chung, K. -S., W. J. Elisens, and J. J. Skvarla. 2010. Pollen morphology and its phylogenetic significance in tribe Sanguisorbeae (Rosaceae). Plant Systematic and Evolution 285:139-148. doi: 10.1007/s00606-009-0262-9.

Cuyckens, G. A. E., D. A. Christie, A. I. Domic, L. R. Malizia, and D. Renison. 2016. Climate change and the distribution and conservation of the world's highest elevation woodlands in the South American Altiplano. Global and Planetary Change 137:79-87. doi: 10.1016/j.gloplacha.2015.12.010.

Etter, A., and L. A. Villa. 2000. Andean forests and farming systems in part of the Eastern Cordillera (Colombia). Mountain Research and Development 20:236-245. doi: 0.1659/0276-4741(2000)020[0236:AFAFSI]2.0.CO;2.

Fajardo-Gutiérrez, F., J. Infante-Betancour y D. M. Cabrera-Amaya. 2018. Modelización de la distribución potencial del género Polylepis en Colombia y consideraciones para su conservación. Ecología Austral 28:202-215

Fernández, M., M. Mercado, and E. Martínez. 2018. Estructura y composición florística de un fragmento boscoso de Polylepis besserihieron subsp. besseri en Sacha Loma (Cochabamba). Revista Boliviana de Ecología y Conservación Ambiental 9:15-27.

Fjeldså, J., and M. Kessler. 1996. Conserving the biological diversity of Polylepis Woodlands of the highland of Perú and Bolivia. Nordeco, Denmark.

Fjeldså, J., and M. Kessler. 2004. Conservación de la biodiversidad de los bosques de Polylepis de las tierras altas de Bolivia. Una contribución al manejo sustentable de los Andes. FAN, San Cruz de la Sierra, Bolivia.

Gareca, E. E., Y. Y. Martinez, C. Solis y L. F. Aguirre. 2018. Efectos de los árboles exóticos y del ambiente materno sobre la producción de seillas. La germinación y el crecimiento inicial de Polylepis subtusalbida (Rosaceae) en el Parque Nacional Tunari, Bolivia. Ecologia Austral 28:262-277

Harding, P. T., and F. Rose. 1986. Pasture-woodlands in lowland Britain: a review of their importance for wildlife conservation. Natural Environment Research Council, Institute of Terrestrial Ecology, Abbots Ripton, Huntingdon.

Henríquez, C. A., G. J. Sotes, and R. O. Bustamante. 2012. Fenología reproductiva de Pouteria splendens (Sapotaceae). Gayana Botánica 69:251-255. doi: 10.4067/S0717-66432012000200004.

Kerr, M. S. 2004. A phylogenetic and biogeographic analysis of Sanguisorbeae (Rosaceae), with emphasis on the Pleistocene radiation of the high Andean genus Polylepis. PhD Thesis. College Park, University of Maryland.

Kessler, M. 2002. The 'Polylepis problem': where do we stand? Ecotropica 8:97-110.

Kessler, M. 2006. Bosques de Polylepis. Pp: 110-120. in M. Moraes, B. Ollgaard, L.P. Kvist, F. Borchsenius, and H. Balslev (eds.) Botánica Económica de los Andes Centrales. Universidad Mayor de San Andrés, La Paz, Bolivia.

Lanza, M. G., M. P. Chartier y P. I. Marcora. 2018. Relación clima-crecimiento radial de Polylepis australis en un gradiente altitudinal en las sierras grandes de Córdoba, Argentina. Ecología Austral 28:278-290

Leitch, I. J., and M. D. Bennett. 1997. Polyploidy in angiosperms. Trends in Plant Science 2:470-476. doi: 10.1016/S13601385(97)01154-0

López, C. L., A. I. Domic, C. Mayta, E. García y S. C. Gallegos. 2018. Fenología reproductiva de la Queñua (Polylepis incarum Rosaceae) durante un ciclo anual en la puna mesofítica de La Paz, Bolivia. Ecología Austral 28: $301-309$

Montalvo, J., D. Minga, A. Verdugo, J. López, D. Guazhambo, D. Pacheco, D. Siddons, A, Crespo y E. Zárate. 2018. Características morfológico-funcionales, diversidad arbórea, tasa de crecimiento y de secuestro de carbono en especies y ecosistemas de Polylepis del sur de Ecuador. Ecología Austral 28: 249-261

Morales, L. V., B. Fuentealba, C.S. Sevillano, M. I. Gómez, M. C. Segovia-Salcedo, D. Renison, D. Green, C. Aucca e I. Hensen. 2018. Oportunidades para acercar la ciencia a la práctica de la restauración de bosques y arbustales de Polyepis. Ecología Austral 28: 291-300

Muñoz-Schick, M., A. Moreira-Muñoz, and S. Moreira Espinoza. 2012. Origen del nombre de los géneros de plantas vasculares nativas de Chile y su representatividad en Chile y el mundo. Gayana Botánica 69:309-359. doi: 10.4067/ S0717-66432012000200011.

Navarro, G., J. A. Molina, and N. D. Barra. 2005. Classification of the high-Andean Polylepis forests in Bolivia. Plant Ecology 176:113-130. doi: 10.1007/s11258-004-0025-1.

Neto, C., A. Vaisberg, B. Zhou, D. Kingston, and G. Hammond. 2000. Cytotoxic Triterpene Acids from the Peruvian Medicinal Plant Polylepis racemosa. Planta Medica 66: 483-484. doi: 10.1055/s-2000-8583.

Poca, M., A. Cingolani, D.E. Gurvich, J. I. Whitworth-Hulse y V. Saur Palmieri. 2018. La degradación de los bosques de altura del centro de Argentina reduce su capacidad de almacenamiento de agua. Ecología Austral 28: 235-248

Purcell, J., and A. Brelsford. 2004. Reassessing the causes of decline of Polylepis, a tropical subalpine forest. Ecotropica 10: 155-158.

Renison, D., L. Morales, G. A. E. Cuyckens, C. S. Sevillano y D. M. Cabrera- Amaya. 2018. Ecología y conservación de los bosques y arbustales de Polylepis: ¿ Qué sabemos y qué ignoramos?. Ecología Austral 28: 163-174

Rossi, C., I. Galindo, G. Huaman, B. Cuadros, Y. Ortega, E. Quispitupac y N. Martínes. 2018. Primer estudio de la riqueza de coleópteros en un bosque de Polylepis tormentella del distrito de Chaviña (Ayacucho, Perú). Eco Austral 28: $229-234$

Segovia-Salcedo, M. C., A. Domic, T. E. Boza y M. Kessler. 2018. Situación taxonómica de las especies del género de Polylepis. Implicancias para los estudios ecológicos, la conservación y la restauración de sus bosques. Ecología Austral 28: 188-201

Sevillano-Ríos, C. S., A. D. Rodewald y L. V. Morales. 2018. Ecología y conservación de las aves asociadas con Polylepis ¿qué sabemos de esta comunidad cada vez más vulnerable?. Ecología Austral 28: 216-228

Simpson, B. B. 1986. Speciation and specialization of Polylepis in the Andes. Pp. 304-316 in F. Vuilleumier and M. Monasterio (eds.). High-Altitude Tropical Biogeography. Oxford University Press, Oxford, UK.

Soltis, P. S., D. B. Marchant, Y. Van de Peer, and D. E. Soltis. 2015. Polyploidy and genome evolution in plants. Current Opinion in Genetics \& Development 35:119-25. doi: 10.1016/j.gde.2015.11.003.

Southwood, T. R. E. 1961. The number of species of insect associated with various trees. Journal of Animal Ecology 30:1-8. doi: $10.2307 / 2109$.

Vega, C. K., G. Villegas, P. A. Rocabado, J. A. N. Quezada, M. Y. López y A. W. Quevedo. 2018. Biología reproductiva de tres especies de Polylepis ( $P$. neglecta, $P$. incarum y P. pacensis), con énfasis en su comportamiento germinativo. Ecología Austral 28: 310-324

Wendel, J. F. 2015. The wondrous cycles of polyploidy in plants. American Journal of Botany 102:1753-1756. doi: 10.3732/ajb.1500320 